\title{
On the largest Hausdorff compactification of a Hausdorff convergence space
}

\section{Vinod-Kumar}

\begin{abstract}
For a Hausdorff convergence space, necessary and sufficient conditions for its Richardson compactification to be the largest Hausdorff compactification are found and by modifying the convergence structure of the Richardson compactification, it is shown that the largest Hausdorff compactification, whenever it exists, is given by that modified Richardson compactification.
\end{abstract}

\section{Introduction}

In [3] and [4] it is proved that a Hausdorff convergence space has the largest Hausdorff compactification if and only if it has only finitely many nonconvergent ultrafilters. We observe that the proof of the necessity part is not sound and hence one can not say whether or not the same is valid in general. However, we establish its validity in case the largest Hausdorff compactification is given by the Richardson compactification. For the general case, we modify the convergence structure of the Richardson compactification and prove that the largest Hausdorff compactification, whenever it exists, is given by that modified Richardson compactification.

\section{Definitions}

For definitions not given here, the reader is asked to refer to [1] and [6]. Let $X$ be a set. Let $F X$ denote the set of all filters on $X$

Received 26 October 1976. The author wishes to thank Dr Wagish Shukla and Dr Arun K. Srivastava for helpful discussions. 
and $P X$ the set of all subsets of $X$. For $x \in X, \dot{x}=\{A \subseteq X \mid x \in A\}$ is the principal ultrafilter containing $\{x\}$. A convergence structure on $X$ is a function $q_{X}$ from $F X$ to $P X$ satisfying the following conditions :

(1) for $x \in X, x \in q_{X}(\dot{x})$;

(2) if $\varphi, \psi \in F X$ and $\varphi \subseteq \psi$, then $q_{X}(\varphi) \subseteq q_{X}(\psi)$;

(3) if $x \in q_{X}(\varphi)$, then $x \in q_{X}(\varphi \cap \dot{x})$.

The pair $\left(X, q_{X}\right)$ is called a convergence space; $\left(X, q_{X}\right)$ is called Hausdorff if and only if $q_{X}(\varphi)$ is at most a singleton for every $\varphi \in F X$; $A \subseteq X$ is called open in $\left(X, q_{X}\right)$ if and only if $x \in A$ and $x \in q_{X}(\varphi)$ imply that $A \in \varphi$. By a space we shall mean a Hausdorff convergence space and by a compactification a Hausdorff compactification. We will treat an embedding as an inclusion map. Call a space essentially compact if and only if it has only finitely many nonconvergent ultrafilters. A space is called locally compact ([3]) if and only if it is open in each of its compactifications. Let $H$-Conv denote the category of spaces and continuous maps. Let $\mathrm{EH}-\mathrm{CO} v \mathrm{f}$ and $\mathrm{CH}$-COnv denote the subcategories of $H$-Conv consisting of essentially compact spaces and compact spaces respectively. Let $A$ and $B$ be subcategories of $H$-Conv such that $B \subseteq A . B$ is called embedding epireflective in $A$ if and only if $B$ is epireflective in $A$ and each reflection map is an embedding.

Let $\left(X, q_{X}\right)$ be a space. For $A \subseteq X$, define $\hat{A}$ and $A^{*}$ by $\hat{A}=\{\varphi \in E X \mid A \in \varphi$ and $\varphi$ is a nonconvergent ultrafilter $\}$ and $A^{*}=A \cup \hat{A}$. Thus, in particular, $X^{*}=X \cup \hat{X}$. For $\varphi \in E X$ let $\varphi^{\prime}$ be the filter $\left\{K \subseteq X^{*} \mid K \cap X \in \varphi\right\}$ on $X^{*}, \varphi^{0}$ the filter generated by $\{A \cup\{\varphi\} \mid A \in \varphi\}$ on $X^{*}$, and $\varphi^{*}$ the filter generated by $\left\{A^{*} \mid A \in \varphi\right\}$ on $X^{*}$. For $\Phi \in F X^{*}$, let $\Phi_{*}$ be the filter $\left\{A \mid A^{*} \in \Phi\right\}$ on $X$ and $\Phi_{0}$ the set $\{K \cap X \mid K \in \Phi\}$.

Let $f:\left(X, q_{X}\right) \rightarrow\left(Y, q_{Y}\right)$ be a function. For $\varphi \in F X$, let $f \varphi$ be the filter $\left\{B \subseteq Y \mid f^{-1} B \in \varphi\right\}$ on $Y$. Define a function $f^{*}: X^{*} \rightarrow Y^{*}$ as follows; for $x \in X$, 


$$
f^{*} x=f x
$$

and for $\varphi \in \hat{X}$,

$$
f^{*} \varphi= \begin{cases}f \varphi & \text { if } f \varphi \in \hat{Y}, \\ y & \text { if } y \in q_{Y}(f \varphi) .\end{cases}
$$

LEMMA 1. (i) If $\Phi \in F X^{*}$ and $X \in \Phi$, then $\Phi_{0} \in F X, \Phi_{0}=\Phi_{*}$, and $\Phi=\Phi_{0}^{\prime}$.

(ii) If $\Phi \in E X^{*}$ is an ultrafizter and $\varphi \in \hat{X}$, then $\varphi^{0} \subseteq \Phi$ if and only if either $\Phi=\varphi^{\prime}$ or $\Phi=\dot{\varphi}$.

(iii) For $\varphi \in \hat{X}$,

$$
f *_{\varphi}^{0}= \begin{cases}(f \varphi)^{0} & \text { if } f \varphi \in \hat{Y}, \\ \dot{y} \cap(f \varphi)^{\prime} & \text { if } y \in q_{Y}(f \varphi) .\end{cases}
$$

Proof. Obvious.

LEMMA 2. A space $\left(X, q_{X}\right)$ is essentially compact if and only if for every ultrafilter $\Phi \in F X^{*}$, either $\Phi=\dot{\varphi}$ for some $\varphi \in \hat{X}$ or $\Phi=\varphi^{\prime}$ for some $\varphi \in F X$.

Proof. Let $\left(X, q_{X}\right) \in E H-C o n v$. Let $\Phi \in F X^{*}$ be an ultrafilter. Then $\hat{X} \in \Phi$ or $X \in \Phi$. If $\hat{X} \in \Phi, \hat{X}$ being finite, there exists $\varphi \in \hat{X}$ such that $\{\varphi\} \in \Phi$. This implies that $\Phi=\dot{\varphi}$. If $X \in \Phi$, then, by Lemma $1, \Phi_{0} \in F X$ and $\Phi=\Phi_{0}^{\prime}$. Conversely, to prove that $\hat{X}$ is finite, it suffices to prove that if $\alpha \in F \hat{X}$ is an ultrafilter, then there exists $\varphi \in \hat{X}$ such that $\alpha^{\prime}=\dot{\varphi}$, where $\alpha^{\prime}=\left\{K \subseteq X^{*} \mid K \cap \hat{X} \in \alpha\right\}$. But this is obvious because of the given condition and the fact that $\alpha^{\prime} \in F X^{*}$ is an ultrafilter and $X \notin \alpha^{\prime}$.

We shall use the following result of [4].

PROPOSITION 3. If a space has the largest compactification, then it is locally compact.

The above result is proved in [4]. The following is a shorter proof. 
Proof. Let $\left(L X, q_{L X}^{I}\right)$ be the largest compactification of a space $\left(X, q_{X}\right)$. It suffices to prove that $X$ is open in $\left(L X, q_{L X}^{1}\right)$. Let $\left(S X, q_{S X}\right)$ be the one point compactification of $\left(X, q_{X}\right)$ as given in [2]. Then there exists a continuous map $g:\left(L X, q_{L X}^{1}\right) \rightarrow\left(S X, q_{S X}\right)$ such that $g$ is the identity function on $X$. This implies that $X=L X-g^{-1}(S X-X)$ is open in $\left(L X, q_{L X}^{1}\right)$.

Let $\left(X, q_{X}\right)$ be a space. Define a convergence structure $q_{X^{*}}^{0}$ on $X^{*}$ as follows. Let $\Phi \in F X^{*}$. For $x \in X, x \in q_{X^{*}}^{0}(\Phi)$ if and only if $X \in \Phi$ and $x \in q_{X}\left(\Phi_{0}\right)$, and for $\varphi \in \hat{X}, \varphi \in q_{X^{*}}^{0}$ if and only if $\varphi^{0} \subseteq \Phi$

LEMMA 4. (i) $\left(X^{*}, q_{X^{*}}^{0}\right)$ is a space with $\left(X, q_{X}\right)$ as its dense and open subspace.

(ii) $\left(X^{*}, q_{X^{*}}^{0}\right)$ is compact if and only if $\left(X, q_{X}\right)$ is essentially compact.

Proof. (i) is obvious.

(ii) If $\left(X, q_{X}\right) \in E H-C o n v$, then $\left(X^{*}, q_{X^{*}}^{0}\right) \in \mathrm{CH}$-Conv by Lemma 2 . Suppose that $\left(X^{*}, q_{X^{*}}^{0}\right) \in \mathrm{CH}$-Conv. Let $\Phi \in F X^{*}$ be an ultrafilter. Then $\Phi$ is convergent and therefore either $\Phi=\Phi_{0}^{\prime}$ or $\varphi^{0} \subseteq \Phi$ for some $\varphi \in \hat{X}$. In the latter case, by Lemma $1, \Phi=\varphi^{\prime}$ or $\Phi=\dot{\varphi}$. Hence $\left(X, q_{X}\right) \in E H-$ Conv.

Let $f \in E H-\operatorname{Conv}\left(\left(X, q_{X}\right),\left(Y, q_{Y}\right)\right)$. Then $f^{*}:\left(X^{*}, q_{X^{*}}^{0}\right) \rightarrow\left(Y^{*}, q_{Y^{*}}^{0}\right)$ is continuous. The inclusion map $i_{X}:\left(X, q_{X}\right) \rightarrow\left(X^{*}, q_{X^{*}}^{0}\right)$ is a dense embedding. If $\left(Y, q_{Y}\right) \in \mathrm{CH}-\mathrm{Con} v$, then there exists a unique continurus 
$\operatorname{map} g\left(=i_{Y}^{-1} \circ f^{*}\right):\left(X^{*}, q_{X^{*}}^{0}\right) \rightarrow\left(y, q_{Y}\right)$ such that $g \circ i_{X}=f$. Thus we have proved

PROPOSITION 5. CH-COnv is embedding epireflective in EH-COnv.

COROLLARY 6 ([3]). There exists tize largest compactification for every essentially compact space.

COROLLARY 7. Every essentially compact space is locally compact.

Let $\left(X, q_{X}\right)$ be a space. In [5] a convergence structure $q_{X^{*}}^{*}$ on $X^{*}$ is defined as follows. Let $\Phi \in F X^{*}$. For $x \in X, x \in q_{X^{*}}^{*}(\Phi)$ if and only if $x \in q_{X^{\prime}}\left(\Phi_{*}\right)$, and for $\varphi \in \hat{X}, \varphi \in q_{X^{*}}^{*}(\Phi)$ if and only if $\varphi^{*} \subseteq \Phi$. Then $\left(X^{*}, q_{X^{*}}\right)$ becomes the Richardson compactification of $\left(X, q_{X}\right)$.

The following proposition tells when $q_{X^{*}}^{0}=q_{X^{*}}^{*}$ for a space $\left(X, q_{X}\right)$. First we prove a lemma.

LEMMA 8. Let $X$ be a set and $D \subseteq F X$ be a finite set of ultrafilters. Given $\varphi \in D$ and $A \in \varphi$, there exists $B \subseteq A$ such that for $\psi \in D, B \in \psi$ if and only if $\psi=\varphi$.

Proof. Given $\varphi \in D$, there exists $K \in \varphi$ such that for $\psi \in D$, $K \in \psi$ if and only if $\psi=\varphi$. For given $A \in \varphi$, take $B=K \cap A$.

PROPOSITION 9. The following are equivalent:

(i) $\left(X, q_{X}\right)$ is essentially compact;

(ii) for $\varphi \in \hat{X}, \varphi^{*}=\varphi^{0}$ and $X$ is open in $\left(X^{*}, q_{X^{*}}^{*}\right)$;

(iii) $q_{X^{*}}^{0}=q_{X^{*}}^{*}$.

Proof. (i) implies (ii). Let $\varphi \in \hat{X}$ and $A \in \varphi$. By Lemma 8 , there exists $B \subseteq A$ such that $B^{*}=B \cup\{\varphi\}$. Hence $\varphi^{*}=\varphi^{0} . X$ is open in $\left(X^{*}, q_{X^{*}}^{*}\right)$ by Corollary 7 .

(ii) implies (iii). Clearly $q_{X^{*}}^{0} \geq q_{X^{*}}^{*}$. Let $x \in q_{X^{*}}^{*}(\Phi)$. $X$ being open in $\left(X^{*}, q_{X^{*}}^{*}\right), X \in \varphi$, and so $x \in q_{X^{*}}^{0}(\Phi)$. If $\varphi \in q_{X^{*}}^{*}(\Phi)$, then 
obviously $\varphi \in q_{X^{*}}^{0}(\Phi)$

(iii) implies $(i)$. Since $q_{X^{*}}^{*}$ is compact and $q_{X^{*}}^{0}=q_{X^{*}}^{*}$, $\left(X, q_{X}\right) \in E H-C O n v$ by Lemma 4.

Let $\left(X, q_{X}\right)$ be a space. We define another convergence structure $q_{X^{*}}$ on $X^{*}$ as follows. Let $\Phi \in F X^{*}$. For $x \in X, x \in q_{X^{*}}(\Phi)$ if and only if $x \in q_{X^{\prime}}\left(\Phi_{*}\right)$ and for $\varphi \in \hat{X}, \varphi \in q_{X^{*}}(\Phi)$ if and only if there exists an ultrafilter $\Psi \in F X^{*}$ such that $\varphi^{*} \subseteq \Psi$ and $\dot{\varphi} \cap \Psi \subseteq \Phi$. Observe that $\left(X^{*}, q_{X^{*}}\right)$ is a compactification of $\left(X, q_{X^{\prime}}\right)$. As $q_{X^{*}}$ is a modification of $q_{X^{*}}^{*}$, we shall refer to $\left(X^{*}, q_{X^{*}}\right)$ as the modified Richardson compactification of $\left(X, q_{X}\right)$.

LEMMA 10. If $q_{X^{*}}^{1}$ and $q_{X^{*}}^{2}$ are two convergence structures on $X^{*}$ such that $\left(X, q_{X}\right)$ is a subspace of both $\left(X^{*}, q_{X^{*}}^{1}\right)$ and $\left(X^{*}, q_{X^{*}}^{2}\right)$ and for $\varphi \in \hat{X}, \varphi \in q_{X^{*}}^{I}\left(\varphi^{\prime}\right)$ and $\varphi \in q_{X^{*}}^{2}\left(\varphi^{\prime}\right)$, then a continuous map $g:\left(X^{*}, q_{X^{*}}^{I}\right) \rightarrow\left(X^{*}, q_{X^{*}}^{2}\right)$ such that $g$ is the identity function on $X$, is the identity function on $X^{*}$.

Proof. Obvious.

LEMMA 11. If $\left(L X, q_{L X}^{I}\right)$ is the largest compactification of a space $\left(X, q_{X}\right)$, then $\operatorname{card} L X=\operatorname{card} X^{*}$.

Proof. Let $i_{X}:\left(X, q_{X}\right) \rightarrow\left(L X, q_{L X}^{1}\right)$ be the inclusion map. Define a function $h: X^{*} \rightarrow L X$ as follows. For $x \in X, h x=x$ and for $\varphi \in \hat{X}, h_{\varphi}=t$, where $t \in q_{L X}\left(i_{X} \varphi\right)$. Let $t \in L X-X$. There exists an ultrafilter $\alpha \in F L X$ such that $X \in \alpha$ and $t \in q_{L X}(\alpha)$. Let $\varphi=\alpha_{0}$, where $\alpha_{0}=\{A \subseteq X \mid A \in \alpha\}$. Since $i_{X}=\alpha, \varphi \in \hat{X}$. By definition of $h, h \varphi=t$ implying that $h$ is onto. $\left(L X, q_{L X}^{l}\right)$ being the largest 
compactification of $\left(X, q_{X}\right)$, there exists a continuous map $g:\left(L X, q_{L X}^{\perp}\right) \rightarrow\left(X^{*}, q_{X^{*}}\right)$ such that $g \circ i_{X}$ is the identity function. Now $h$ is one-to-one because $g \circ h$ is the identity function. We shall identify $i_{X} \varphi$ and $\varphi^{\prime}$, and $t \in q_{L X}\left(i_{X} \varphi\right)$ and $\varphi$, for $\varphi \in \hat{X}$.

THEOREM 12. The Zargest compactification of a space, whenever it exists, is given by the modified Richardson compactification.

Proof. If $\left(L X, q_{L X}^{\perp}\right)$ is the largest compactification of a space $\left(X, q_{X}\right)$, then, by Lemma 11 , card $L X=\operatorname{card} X^{*}$. Thus $\left(L X, q_{L X}^{I}\right)$ can be rewritten as $\left(X^{*}, q_{X^{*}}^{1}\right)$. There exists a continuous map $g:\left(X^{*}, q_{X^{*}}^{I}\right) \rightarrow\left(X^{*}, q_{X^{*}}\right)$ such that $g$ is the identity function on $X$. By Lemma $10, g$ is the identity function on $X^{*}$; hence $q_{X^{*}}^{1} \geq q_{X^{*}}$. $\left(X, q_{X}\right)$ being locally compact by Proposition 3, $x \in q_{X^{*}}(\Phi)$ implies that $x \in q_{X^{*}}^{1}(\Phi)$. Now to show that $q_{X^{*}} \geq q_{X^{*}}^{1}$, it suffices to prove that if $\varphi \in q_{X^{*}}(\Phi)$ for an ultrafilter $\Phi \in F X^{*}$, then $\varphi \in q_{X^{*}}^{1}(\Phi)$, which is obvious, since $\left(X^{*}, q_{X^{*}}^{1}\right)$ is compact and $g$ is the identity function on $X^{*}$.

The following theorem is a categorical version of Theorem 12 .

THEOREM 12'. If $A$ is a full subcategory of $\mathrm{H}$-Conv such that $\mathrm{CH}$-Conv is embedding epireflective in A, then the epireflection is given by the modified Richardson compactification.

Now we give relations between $q_{X^{*}}^{0}$ and $q_{X^{*}}$, and $q_{X^{*}}^{*}$ and $q_{X^{*}}$. PROPOSITION 13. Let $\left(X, q_{X}\right)$ be a space.

(i) $q_{X^{*}}=q_{X^{*}}^{0}$ iff $\left(X, q_{X}\right)$ is essentially compact. 
(ii) $q_{X^{*}}=q_{X^{*}}^{*}$ iff $\varphi^{0}=\varphi^{*}$ for every $\varphi \in \hat{X}$.

Proof. (i) is obvious in view of Lemma 4 and Theorem 12.

(ii). Let $q_{X^{*}}=q_{X^{*}}^{*}$. Let $\varphi \in \hat{X}$. Since $\varphi \in q_{X^{*}}^{*}\left(\varphi^{*}\right)$, there exists an ultrafilter $\Phi \in F X^{*}$ such that $\varphi^{*} \subseteq \Phi$ and $\dot{\varphi} \cap \Phi \subseteq \varphi^{*}$. Hence $\varphi^{0}=\varphi^{*}$. The converse is obvious.

THEOREM 14. The Richardson compactification of a space is the largest compactification iff the space is essentially compact.

Proof. Let $\left(X, q_{X}\right) \in E H-C o n v$. By Proposition 9, $q_{X^{*}}^{0}=q_{X^{*}}^{*}$. Hence $\left(X^{*}, q_{X^{*}}^{*}\right)$ is the largest compactification of $\left(X, q_{X}\right)$. In case $\left(X^{*}, q_{X^{*}}^{*}\right)$ is the largest compactification of $\left(X, q_{X}\right), q_{X^{*}}=q_{X^{*}}^{*}$ by Theorem 12. Now $\varphi^{0}=\varphi^{*}$ and $X$ is open in $\left(X^{*}, q_{X^{*}}^{*}\right)$. Hence $\left(X, q_{X}\right) \in E H-\operatorname{Conv}$.

COROLLARY 15. EH-Conv is the Zargest full subcategory of H-Conv such that the Richardson compactification defines an epireflection from EH-Conv to CH-Conv.

COROLLARY 16. The Richardson compactification $\left(X^{*}, q_{X^{*}}^{*}\right)$ of a space $\left(X, q_{X}\right)$ is the largest compactification iff

(i) $\left(X, q_{X}\right)$ is locally compact, and

(ii) $\dot{\varphi} \cap \varphi^{\prime}=\varphi^{*}$ for every $\varphi \in \hat{X}$.

The proof of the above result given in [4] is not sound.

\section{References}

[1] D.C. Kent, "Convergence quotient maps", Fund. Math. 65 (1969), 197-205.

[2] C.J.M. Rao, "On smallest compactification for convergence spaces", Proc. Amer. Math. Soc. 44 (1974), 225-230. 
[3] C.J.M. Rao, "On largest Hausdorff compactification for convergence spaces", Bul2. Austral. Math. Soc. 12 (1975), 73-79.

[4] Challa Jagan Mohan Rao, On extremal compactifications of convergence spaces (Department of Mathematics, Indian Institute of Technology Kanpur, Kanpur, 1975).

[5] G.D. Richardson, "A Stone-Čech compactification for limit spaces", Proc. Amer. Math. Soc. 25 (1970), 403-404.

[6] G.D. Richardson and D.C. Kent, "Regular compactifications of convergence spaces", Proc. Amer. Math. Soc. 31 (1972), 571-573.

Department of Mathematics, Indian Institute of Technology, Hauz Khas, New Delhi, India. 